\section{The clinical abnormalities used by primary care practitioners to direct testing for FeLV and FIV in the UK}

\section{Jordana March, Nicholas Bexfield, Mark Dunning}

University of Nottingham, Sutton Bonington, Leicestershire, UK

\section{BACKGROUND}

Feline Immunodeficiency Virus (FIV) and Feline Leukaemia Virus (FeLV) have a relatively low prevalence in the UK, but testing is frequently performed. Little is known about which cats are tested by primary care veterinary surgeons, and particularly which signs or results influence the decision to test for these diseases.

\section{OBJECTIVES}

To determine whether the signalment and presenting clinical signs influence primary care veterinary surgeon's decisions to test cats for FeLV or FIV. Moreover, we aimed to determine which signs were associated with a positive diagnosis.

\section{MATERIALS AND METHODS}

Clinical records from four primary care veterinary practices in the UK were reviewed. Two-hundred and seventy-five cats were tested for FeLV/FIV. Information regarding signalment, clinical signs at the time of testing, history and test methodology were collected. To analyse the data, clinical signs and historical findings were assigned to categories based on body systems, but where this was not possible, a category of 'non-specific' was created. Data were analysed for all cases. Cases that tested positive for FeLV or FIV, and cases that tested negative were identified, and data were analysed and compared between these categories.

\section{RESULTS}

Out of the 275 cats tested for FeLV/FIV, 4.4\% of cats were positive for FIV and $1.8 \%$ were positive for FeLV. Signalment did not influence which cats were tested, although more male cats (58.8\%) were positive for either FeLV or FIV. Cats with signs in the 'non-specific' category were most frequently tested (121 cats), followed by those with signs relating to the gastrointestinal (Gl), respiratory and oral systems (81, 56 and 52 cats, respectively). Neoplasia and haematological dyscrasias were more frequently encountered in cases positive for FeLV or FIV than cases that were negative for either disease $(5.9 \%$ versus $0.4 \%$ for neoplasia, 35.3\% versus $8.9 \%$ for haematological dyscrasias).

\section{CONCLUSIONS}

The majority of veterinary surgeons use non-specific clinical signs to direct testing for FeLV and FIV which results in predominantly negative results $(95.6 \%$ for FIV and $98.2 \%$ for FeLV). This current approach to testing suggests clinicians would gain most value in using FeLV and FIV tests as screening tests to rule out both diseases, given the low prevalence. If attempting to use the tests in a confirmatory way, cats with signs related to the $\mathrm{Gl}$, oral and respiratory systems along with neoplastic disease or haematological dyscrasias should be selected.

\section{What is the best method of estimating energy intake for weight loss in obese dogs? \\ Hannah Sargent ${ }^{1}$, Georgiana Woods ${ }^{2}$, Vincent Biourge ${ }^{3}$, Alex German ${ }^{2}$}

\author{
1 University of Nottingham, Nottingham, UK \\ 2 University of Liverpool, Liverpool, UK \\ 3 Royal Canin Research Centre, Aimargues, France
}

The main therapeutic approach for canine obesity is restricting caloric intake, this succeeds provided that the energy intake (El) during weight loss is less than the dog's energy expenditure. Many methods exist for predicting the starting El required for weight loss in obese dogs. Methods can incorporate either resting energy requirement (RER = $70 \mathrm{kcal} / \mathrm{kg}^{0.75} /$ day) or maintenance energy requirement (MER $=105 \mathrm{kcal} / \mathrm{kg}^{0.75} /$ day), and be based upon either starting body weight (SBW) or target body weight (TBW, estimated from body condition score). In addition, some methods make adjustments for sex and neuter status.

In this retrospective study, the performance of predictive equations were assessed:

- Equation 1 (Eq1). El = $0.80 \times \mathrm{RER} /$ day, using SBW.
Equation 2 (Eq2). El = $0.80 \times \mathrm{RER} /$ day, using TBW. Equation 3 (Eq3). El $=0.60 \times \mathrm{MER} /$ day, using TBW. - Equation 4 (Eq4). El $=0.55-0.65 \times \mathrm{MER}$, again using TBW, with the coefficient differing by sex and neuter status (entire male 0.65; entire female or neutered male 0.60 ; neutered female 0.55 ).

Predictions were compared with retrospective weight loss data from 74 obese dogs that had attended the Royal Canin Weight Management Clinic, University of Liverpool. All dogs had successfully reached TBW, and rate of weight loss had been $>0.5 \% /$ week in the first 28 days. Associations were assessed with simple linear regression, whilst BlandAltman plots were used to determine accuracy.

For all equations, there was strong positive correlation with actual El (R2 $=0.93-0.97, P<0.001$ for all). On average, Eq1 over-estimated actual El (median $+13 \%$; $-26 \%$ to $+82 \%$, $P<0.001$ ), with only $27 / 74$ (36\%) of estimates being within $\pm 10 \%$. Eq2 under-estimated actual El on average (median $-6 \%,-32 \%$ to $+42 \%, P<0.001)$, although results were more accurate than for Eq1 (44/74 [59\%] of estimates within $\pm 10 \%$ ). Eq3 over-estimated actual El on average (median $6 \%,-23 \%$ to $+59 \%, P<0.001$ ), but its accuracy was marginally better than Eq2 (49/74 [66\%] within $\pm 10 \%)$. Eq4 performed best of all, with average results not being significantly different from actual El (median $2 \%,-19 \%$ to $46 \%, P=0.089$ ), 